\title{
Electric Motor Modeling for Conceptual Aircraft Design
}

\author{
Robert A. McDonald* \\ California Polytechnic State University, San Luis Obispo, CA, 93407
}

\begin{abstract}
Electric motors have achieved very high efficiency and reliability; and in some cases, very high specific power. As the energy storage capabilities of batteries and fuel cells advance, these technologies are increasingly being considered for aircraft primary propulsion. In order to design electric aircraft, aircraft designers must be able to understand and model the performance characteristics of electric motors. Most electric machine modeling techniques either ignore potentially important phenomena or require an extreme level of detail undesirable for conceptual aircraft design. In this paper, a compromise approach is proposed which can capture the salient details of motor performance without requiring intricate modeling of the electric machine.
\end{abstract}

\section{Introduction}

$A^{\mathrm{N}}$ engine deck is an essential tool for modeling aircraft performance for design. An engine deck models Athrust lapse and part-power behavior of an engine by providing thrust and fuel (energy) consumption as a function of speed, altitude, and throttle setting.

An engine deck for an electric prime mover can be built by coupling an electric motor model to an appropriate propulsor such as a propeller or a duct/fan combination. Representing the full-throttle lapse behavior of the electric propulsor requires an electric motor model which includes the operating constraints/limits for the motor. Representing the energy consumption of the electric propulsor requires an electric motor model which includes the part-power efficiency of the motor.

At its most abstract, an electric motor is a device which converts electric power $P_{i n}$ to mechanical shaft power $P_{\text {shaft }}$. The primary metric of an electric motor is the efficiency ratio of output to input power $\eta=P_{\text {shaft }} / P_{\text {in }}$. Equally critical is the form of the power. Mechanical shaft power is the product of the torque, $Q$, and the rotational speed, $\omega$, i.e. $P_{\text {shaft }}=\omega Q$. Likewise the electrical power for a DC motor (for example) is the product of the current, $I$, and potential drop across the motor, $V$, i.e. $P_{i n}=I V$. A motor's operating limits are most commonly specified as limits on these inputs or outputs; for example, maximum power, maximum torque, maximum speed, or maximum current.

When modeling a motor as a component in a greater system, it makes sense to focus on this relationship between inputs and outputs. A motor's performance may be considered as a forward problem where the motor efficiency or output is a function of the motor input and some other operating conditions, i.e. $\left[\eta\right.$ or $\left.P_{\text {shaft }}\right]=f\left(P_{i n}\right.$ or $\left.I, V\right)$. Alternatively, a motor may be considered as an inverse problem where the motor efficiency or input is a function of the motor output and some other operating conditions, i.e. $\left[\eta\right.$ or $\left.P_{\text {in }}\right]=f\left(P_{\text {shaft }}\right.$ or $\left.\omega, Q\right)$. When matching a motor to a mechanical load, the inverse approach is often more useful. The other operating conditions mentioned previously include other state and environmental variables such as motor temperature and ambient air density.

Together, the motor operating envelope and its part-power efficiency behavior constitute a motor map. Motor manufacturers may include a motor map like the one in Figure 1 in a specification sheet describing a motor. ${ }^{1}$

The motor map in Figure 1 represents an inverse perspective on the motor's performance; the motor efficiency may be read from the contours as a function of the $x$ and $y$ axes (speed and torque) i.e. $\eta=f(\omega, Q)$. The motor operating limits are indicated by the axis limits and the bold black line.

\footnotetext{
*Associate Professor, Aerospace Engineering, One Grand Avenue, AIAA Senior Member.
} 


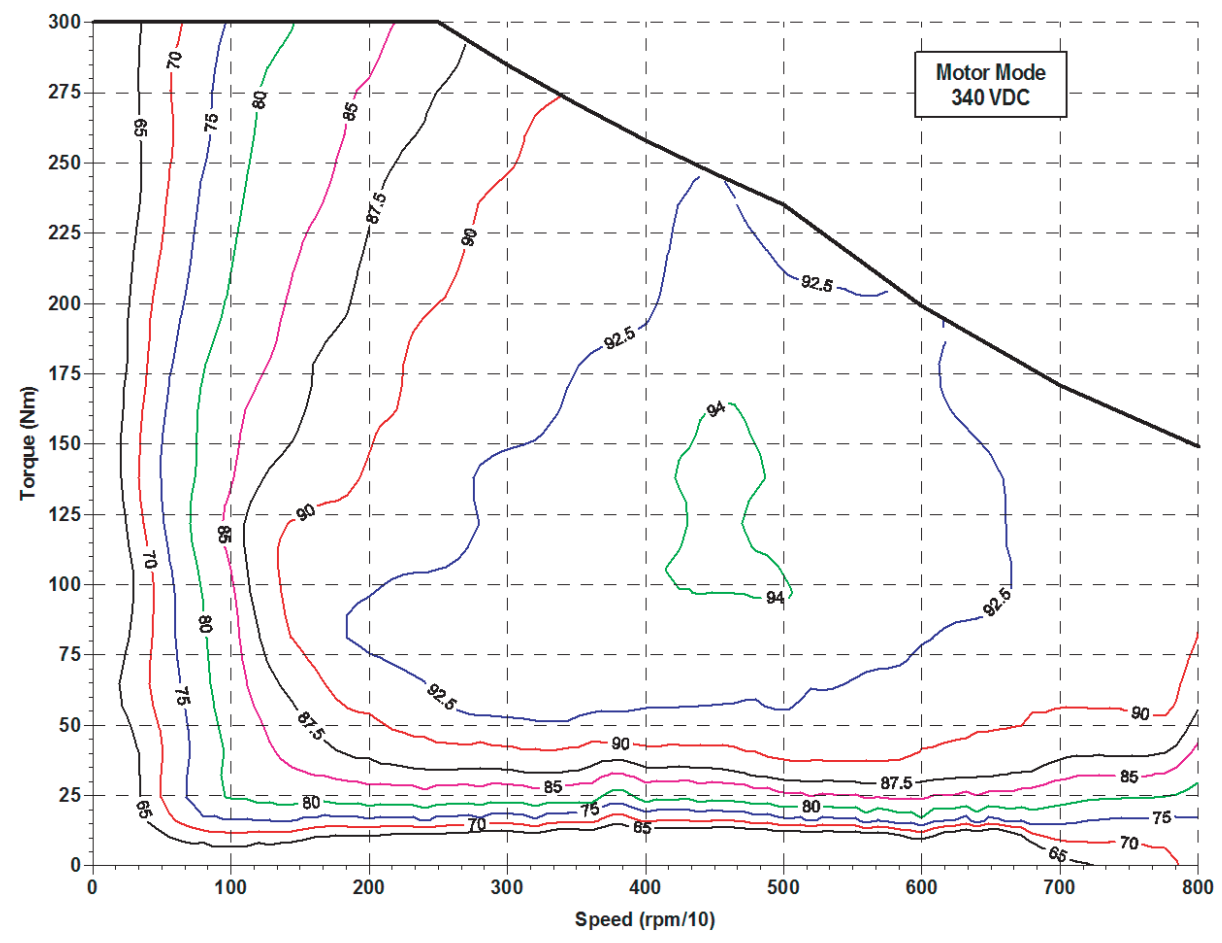

Figure 1. Published motor map for UQM PowerPhase $125 .^{1}$

In addition to representing the performance of a particular motor, Figure 1 exemplifies several characteristics typical of electric motor performance. First, the stall (zero speed, finite torque) efficiency of an electric motor is zero. Likewise, the no-load (finite speed, zero torque) efficiency of an electric motor is also zero. Motor efficiency contours become very steep and go to zero as either speed or torque approach zero. In addition, Figure 1 exhibits a peak efficiency of greater than $94 \%$ near 4,500 rpm and $125 \mathrm{Nm}$ of torque. As for low speed and torque, efficiency for this motor drops off as speed or torque increases from this peak. The existence of an efficiency peak leads to closed contour islands on the efficiency map.

An empirically derived motor map in the form of Figure 1 does not constitute a motor model appropriate for creating an engine deck. The oscillations in the efficiency contours would present significant challenges to an otherwise straightforward interpolation approach. Similarly, any later analysis to find an optimal engine or aircraft operating point will face significant challenges. The author can find no physical justification for the oscillations in these contours. It is believed that these oscillations are an artifact of the experimental error and the process of obtaining smooth contours from inherently sparse and noisy data.

The primary aim of this paper is to present a method through which a map like Figure 1 can be approximated by a motor model appropriate for use in the conceptual aircraft design process.

\section{Background}

There are two main approaches to electric motor and controller modeling - the power loss approach and the equivalent circuit approach.

The power loss approach attempts to build up each of the physical power losses within the electric motor and controller. Some of these losses are modeled according to their fundamental physics while others are approximated from experience or empirical models. The power loss approach is the best option for modeling electric machines which have not yet been built - such as during the design process of a new motor.

The equivalent circuit approach replaces the electric motor and controller with a simplified circuit made of familiar electronic components. The parameters of these components are selected such that the equivalent circuit performs like the motor. In some of these models, the circuit parameters can be readily measured. The equivalent circuit approach has been broadly adopted by the small brushless DC motor industry that 
typically serves the radio control aircraft and small UAV markets.

\section{II.A. Loss Build-up Modeling}

The electric machine community typically attempts to build-up the losses in a particular motor. These include copper loss, core or iron loss, mechanical loss, stray load loss, and controller loss. The copper and controller loss can be readily estimated or measured in the lab. Whereas the mechanical loss (friction and windage) is usually obtained empirically from a no-load current test or a spindown test. The stray load loss captures all unaccounted losses; its estimate is nearly always empirical in origin.

The core or iron loss is the most difficult to model; predicting and minimizing it is also a primary focus of electric machine designers. High-fidelity models involve time-accurate finite element modeling of the magnetic fields within the motor. This requires very detailed knowledge of the design of the motor. Analytical and empirical approaches for modeling these losses also exist, but in all cases this kind of modeling is beyond the scope of the conceptual aircraft design process.

Manufacturers seldom publish loss build-up information sufficient to produce a motor map appropriate for aircraft design work. In the situation where a manufacturer provides an empirical motor map like Figure 1, there is no clear path to constructing a loss buildup model of the motor.

In the spirit of constructing an inverse model of motor performance, it is useful to consider each loss source as a function of motor output, i.e. $P_{L}=f(\omega, Q)$. Different authors give slightly different forms for some of the loss sources; here we will use the forms presented by Larminie and Lowry. ${ }^{2}$

The mechanical losses are a function of motor speed. Friction loss is proportional to motor speed, $P_{f} \propto \omega$, and windage loss is generally considered to be proportional to motor speed cubed, $P_{w} \propto \omega^{3}$.

Copper loss is due to resistive loss in the motor windings and is consequently proportional to current squared. Motor torque is generally proportional to current, so copper losses are proportional to torque squared, $P_{\mathrm{Cu}} \propto Q^{2}$.

Iron loss includes both eddy current loss and hysteresis loss in the motor. These losses are proportional to the frequency of the magnetic flux change in the motor. The frequency is proportional to the motor speed, so iron losses are proportional to motor speed, $P_{\mathrm{Fe}} \propto \omega$.

In constructing their total loss model, Larminie and Lowry also include a constant parasitic loss term, $P_{0}$. The resulting loss model is $P_{L}=P_{f}+P_{w}+P_{\mathrm{Cu}}+P_{\mathrm{Fe}}+P_{0}$. This model can be written as Equation 1 with $C_{i}$ serving as the constants of proportionality for each loss term.

$$
P_{L}=C_{0}+C_{1} \omega+C_{2} \omega^{3}+C_{3} Q^{2}
$$

The analysis of Appendix A reveals that a loss buildup model in the form of Equation 1 is capable of representing a motor map with efficiency islands like those in Figure 1. In addition, at zero speed and torque, power loss is still finite $\left(P_{L}=C_{0}\right)$, which will ensure zero efficiency for this model in stalled and no-load conditions.

\section{II.B. Equivalent Circuit Modeling}

The radio control community has popularized and made effective use of simple equivalent circuit models for brushless DC motors. The standard model employs three parameters, the voltage/speed constant $K_{v}$, the internal resistance $R_{i}$, and the no-load current, $I_{0}$. All reputable motor manufacturers in this market publish the three parameters for the standard model.

The standard model has been employed by the aerospace community. ${ }^{3,4}$ Despite its popularity, the standard model has several weaknesses which some authors have attempted to address - typically in unpublished work. $^{5,6}$

The standard equivalent circuit model may be readily used as an inverse motor model giving $\eta=f(\omega, Q)$. First, the useful voltage is calculated as in Equation 2 from $K_{v}$ and the motor speed.

$$
V_{u}=\frac{\omega}{K_{v}}
$$

Next, the useful current is calculated as in Equation 3 from the shaft power load and the useful voltage; recall that $P_{\text {shaft }}=\omega Q$.

$$
I_{u}=\frac{P_{\text {shaft }}}{V_{u}}
$$


Then the total current is calculated as the sum of the no-load current and the useful current, i.e. $I=$ $I_{0}+I_{u}$, and the total voltage is calculated as the sum of the useful voltage and the voltage drop across the internal resistance, i.e. $V=V_{u}+I R_{i}$.

The input electrical power, $P_{\text {in }}=I V$, and the motor efficiency $\eta=P_{\text {shaft }} / P_{\text {in }}$ may then be readily calculated. When using the standard equivalent circuit model as an inverse motor model, it is generally best to follow each of the steps beginning with Equation 2. However, it can be illuminating to instead expand these relations into a loss equation as a function of motor output, i.e. $P_{L}=f(\omega, Q)$.

The power loss for the equivalent circuit model can be considered as the sum of the no-load current loss and the internal resistance loss, $P_{L}=P_{I_{0}}+P_{R_{i}}$.

The no-load current loss is the product of the no-load current and the useful voltage, $P_{I_{0}}=I_{0} V_{u}$. Substituting Equation 2 into this relation gives Equation 4 for the no-load current loss. Note that the no-load current loss is proportional to motor speed.

$$
P_{I_{0}}=\frac{I_{0}}{K_{v}} \omega
$$

The loss due to internal resistance is the product of the internal resistance and the current squared, $P_{R_{i}}=R_{i} I^{2}$. Substituting Equation 3 into the total current and then Equation 2 into the resulting expression gives Equation 5 after recognizing the shaft power divided by the motor speed as torque.

$$
I=I_{0}+K_{v} Q
$$

After expanding the square, the product of the internal resistance and Equation 5 squared gives Equation 6 for the loss due to internal resistance.

$$
P_{R_{i}}=R_{i} I_{0}{ }^{2}+2 R_{i} I_{0} K_{v} Q+R_{i} K_{v}{ }^{2} Q^{2}
$$

The sum of Equations 4 and 6 gives Equation 7 for the total power loss of an electric motor using the standard equivalent circuit model.

$$
P_{L}=R_{i} I_{0}{ }^{2}+\frac{I_{0}}{K_{v}} \omega+2 R_{i} I_{0} K_{v} Q+R_{i} K_{v}{ }^{2} Q^{2}
$$

The analysis of Appendix A reveals that the standard equivalent circuit model in the form of Equation 7 is not capable of representing a motor map with efficiency islands like those in Figure 1. However, at zero speed and torque, power loss is still finite $\left(P_{L}=R_{i} I_{0}{ }^{2}\right)$, which will ensure zero efficiency for this model in stalled and no-load conditions.

\section{Positive Polynomial Loss Model}

Rather than base a motor model on an equivalent circuit or a buildup of simplified physical loss sources, this work proposes to simply fit a surface to motor performance data. This data can take the form of a published motor map like Figure 1, or it could come from unprocessed motor test stand data. Though it may be intuitive to fit a model to efficiency, best results have been obtained by fitting the power loss; fitting power loss allows some knowledge of the behavior of the system to be built into the functional form. The curve to be fit is chosen to be a polynomial in torque and motor speed; this polynomial is restricted to have only positive (or zero) coefficients.

Restricting the power loss model to non-negative coefficients ensures that no region of the motor operating domain (positive torque and speed) can ever have a negative power loss - efficiency can never be greater than one. If each term in the polynomial is interpreted as an individual loss source, then no loss source can ever become a gain. Including a positive constant term in the polynomial ensures that power loss is always finite and positive - even when torque or speed are zero. Consequently, motor efficiency will always go to zero for the stalled (zero speed) or no-load (zero torque) motor conditions.

Although the polynomial is restricted to positive coefficients and must include a constant term, the number of terms, order of each term and maximum order of the polynomial can be selected by the particular needs of the fit. The analysis of Appendix A establishes restrictions on the minimum order of the loss model to ensure that it is capable of representing islands of efficiency. In order for a positive polynomial power loss model to be able to represent efficiency islands in the operating domain, there must be terms with non-zero 
coefficients of at least second order in speed and torque and also at least one term with non-zero coefficient of at least third combined order

A generic positive polynomial power loss model can be put in the form of Equation 8. Equation 8 is a sum of polynomial terms up to order $n$ in $Q$ and order $m$ in $\omega$. The coefficient for each term, $C_{i, j}$, may be zero or positive, but may not be negative. The $i=0$ terms in the sum are only functions of $\omega$ while the $j=0$ terms are only functions of $Q$. The $i=0, j=0$ term is a constant.

$$
P_{L}=\sum_{i=0}^{n} \sum_{j=0}^{m} C_{i, j} Q^{i} \omega^{j} ; \quad \text { Where } C_{i, j} \geq 0
$$

A broad class of power loss models can be put in the form of Equation 8. The loss buildup according to Larminie and Lowry presented in Section II.A could be represented by Equation 8 with $n=2$ and $m=3$. Similarly, the standard equivalent circuit model presented in Section II.B could be represented by Equation 8 with $n=2$ and $m=1$. In each of these cases, a significant number of the coefficients for terms which do not appear would equal zero.

The data represented in Figure 1 must be placed in terms of power loss (instead of efficiency) before it can be used to fit Equation 8. The motor efficiency is simply the output power divided by the input power, where the input power can be put in terms of shaft power and power loss as in Equation 9.

$$
\eta=\frac{P_{\text {shaft }}}{P_{\text {shaft }}+P_{L}}
$$

Equation 9 can be re-arranged to give the power loss as Equation 10 in terms of shaft power and efficiency. These quantities may be calculated from data points picked from a motor map such as Figure 1.

$$
P_{L}=P_{\text {shaft }} \frac{1-\eta}{\eta}
$$

Using a traditional least squares approach to determine the $C_{i, j}$ coefficients would result in the best possible fit of the data, but it would possibly violate the non-negative restriction on $C_{i, j}$. Instead, a nonnegative least squares (NNLS ${ }^{7}$ algorithm is used which finds the best fit to the data subject to a positivity constraint on all of the coefficients. The NNLS algorithm finds popular use in the astronomical image processing community resulting in good availability of off-the-shelf implementations. Source code implementing the NNLS algorithm is readily available in C, Fortran 77 , and Fortran 90 . The examples in this work were computed using the built-in Matlab function lsqnonneg; older versions of Matlab included the built-in function nnls.

\section{Example}

In this example, the efficiency contours from Figure 1 were digitized and the resulting data was fit to a positive polynomial power loss model. The complete set of digitized points are depicted in Figure 2 as grey circles and black squares. Using all of this data biased the surface fit to the low speed and low torque regions of the operating envelope, which resulted in relatively poor performance capturing the rest of the envelope including the efficiency peak. Instead, only a subset of the data was used for the fit; the results presented in this paper were generated by fitting the surface to only the black squares in Figure 2.

For this example, the terms depicted in Equation 11 were used to fit the power loss; the NNLS fit resulted in non-zero coefficients for each of these terms. No physical or rigorous justification is given for the selection of these terms or the omission of others; this is the set of terms which the author found gave acceptable results in this case.

$$
P_{L}=C_{0,0}+C_{0,1} \omega+C_{2,0} Q^{2}+C_{3,0} Q^{3}+C_{0,3} \omega^{3}+C_{1,3} Q \omega^{3}+C_{3,3} Q^{3} \omega^{3}
$$

The motor model resulting from the NNLS fit of the black squares in Figure 2 to Equation 11 is presented in Figure 3. The fit model provides a useful approximation of the published motor map. The oscillatory behavior of the efficiency contours is smoothed. The asymptotic behavior of efficiency at stalled or no-load condition is correct. The efficiency islands are captured in a reasonable manner. The motor model depicted in Figure 3 would be appropriate for matching to a propulsor to create an engine deck. If the model of the propulsor was sufficiently smooth, then the resulting deck would be appropriate for a wide range of propulsion system or aircraft performance optimization studies. 


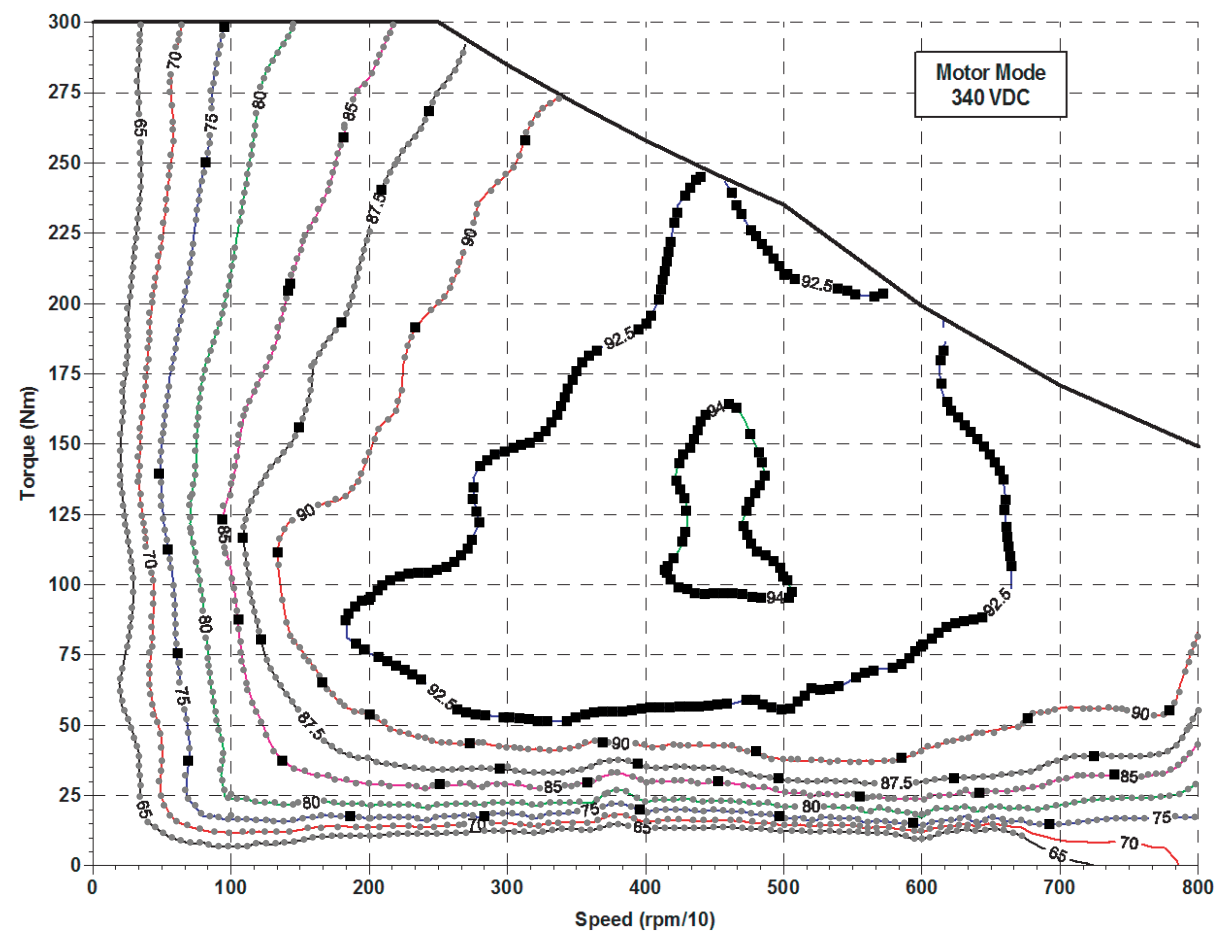

Figure 2. Digitized data from published motor map. The grey circles represent all digitized points. The black squares represent the subset used to fit the motor map.

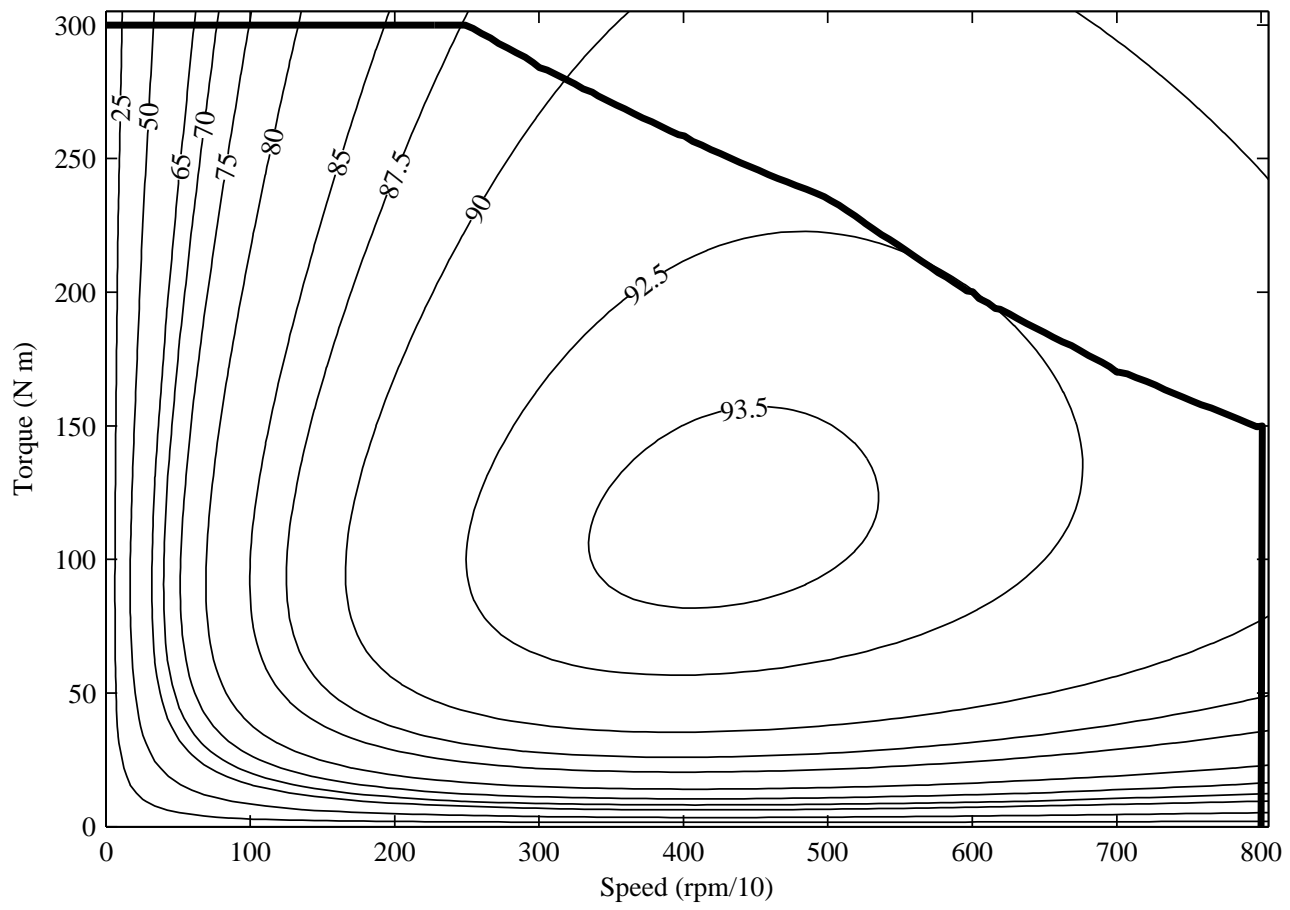

Figure 3. Fit motor map. 
Figure 4 depicts the fit motor model of Figure 3 overlaid on the original manufacturer's motor map from Figure 1 for comparison. Although the fit motor model does not quite capture the peak efficiency of the motor, the model is accurate enough for use in the conceptual aircraft design process.

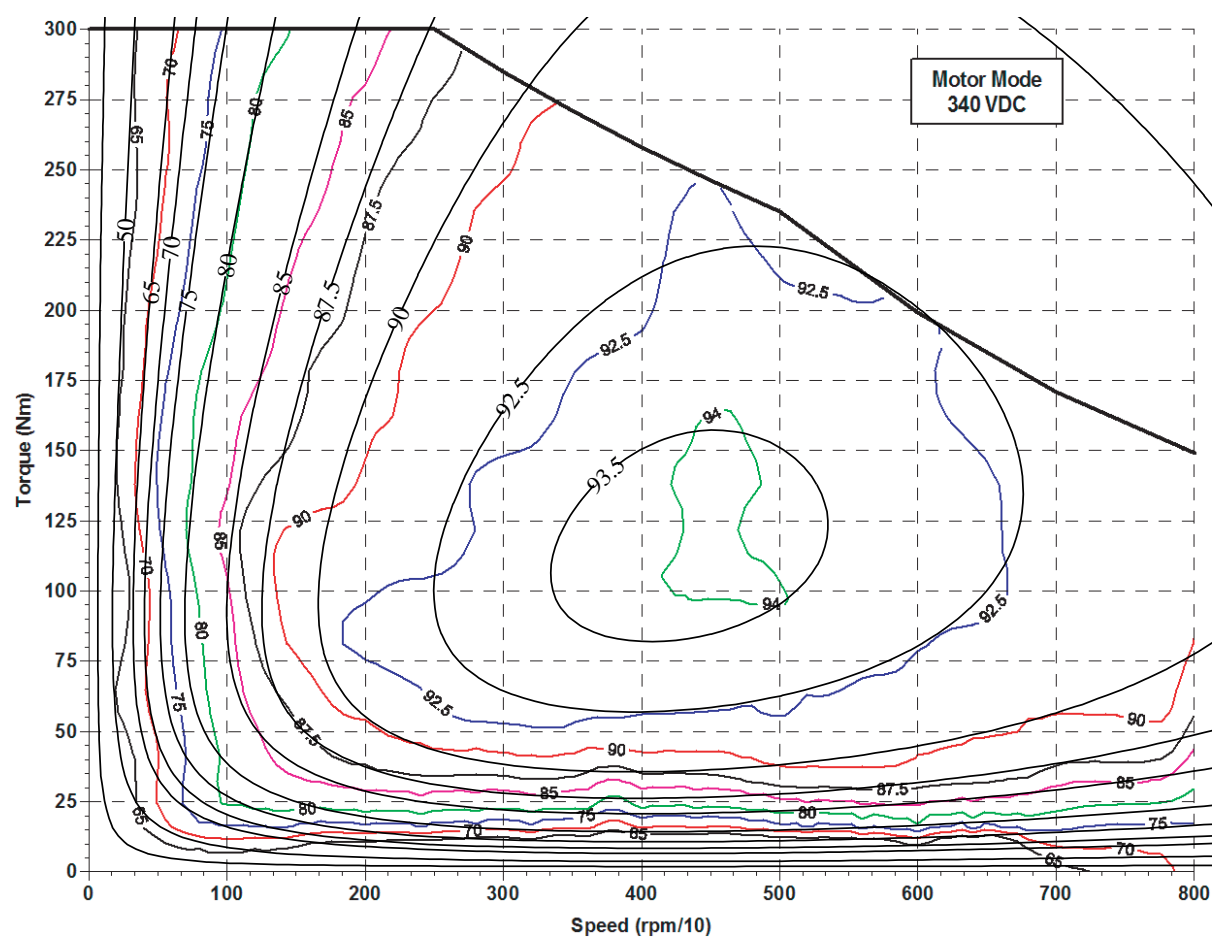

Figure 4. Fit motor map comparison.

\section{Conclusions}

A straightforward means of constructing an approximate model of motor efficiency starting from a published motor map is presented. The use of a positive polynomial to represent power loss is proposed and is shown to be a generalization of two established motor modeling techniques. In the appendix, conditions on a power loss model for representing an efficiency island are derived and applied to a general positive polynomial power loss model. These conditions demonstrate that the standard equivalent circuit model used for brushless DC motors is not able to represent efficiency islands.

As an example, a positive polynomial with some high order terms was fit to data digitized from a motor map published by a manufacturer. The resulting model captured the limiting behavior of an electric motor as well as the efficiency islands that appear within the operating envelope. The model also eliminated spurious oscillations in the motor map resulting in a motor model suitable for generating an electric engine deck and conducting a broad range of conceptual aircraft design studies.

\section{Acknowledgements}

Some of this work was performed while the author served as a member of the Aeronautics Systems Analysis Branch at NASA Langley Research Center, through an Intergovernmental Personnel Act agreement.

\section{A. Efficiency Island Criteria}

Published motor maps like the one included as Figure 1 often exhibit efficiency islands representing the peak operating efficiency of the motor. Depending on the application of a motor model, it may be necessary 
to ensure that a chosen model is capable of representing these efficiency islands. We seek to establish criteria for an inverse motor model in the form $\eta=f(\omega, Q)$ to support efficiency islands.

Recall the motor efficiency, $\eta=P_{\text {shaft }} / P_{\text {in }}$; where the input power can be taken as the sum of the output shaft power and the power loss, i.e. $P_{i n}=P_{\text {shaft }}+P_{L}$. Substituting the mechanical definition for shaft power gives Equation 12 for motor efficiency. If the power loss is understood as a function of the motor output, then Equation 12 is an inverse equation for the motor efficiency.

$$
\eta=\frac{\omega Q}{\omega Q+P_{L}}
$$

Taking derivatives of Equation 12 with respect to speed and torque and setting them equal to zero will allow efficiency island conditions on the power loss equation to be established. First, the partial derivative of Equation 12 with respect to torque gives Equation 13.

$$
\frac{\partial \eta}{\partial Q}=\frac{\omega}{\omega Q+P_{L}}-\frac{\omega Q}{\left(\omega Q+P_{L}\right)^{2}}\left(\omega+\frac{\partial P_{L}}{\partial Q}\right)
$$

Equation 13 set equal to zero can be simplified to yield Equation 14, the first efficiency island condition on the power loss equation.

$$
0=P_{L}-Q \frac{\partial P_{L}}{\partial Q}
$$

Motor speed and torque appear in the same way in Equation 12. Consequently, taking the derivative with respect to motor speed, setting it equal to zero, and simplifying the resulting equation follows the same procedure that lead to Equation 14 and results in Equation 15, the second efficiency island condition on the power loss equation.

$$
0=P_{L}-\omega \frac{\partial P_{L}}{\partial \omega}
$$

In order for an efficiency island to exist, both Equations 14 and 15 must be satisfied by a candidate power loss equation. Furthermore, any combination of Equations 14 and 15 will form an additional condition. Summing Equations 14 and 15 results in Equation 16, the third efficiency island condition on the power loss equation.

$$
0=2 P_{L}-Q \frac{\partial P_{L}}{\partial Q}-\omega \frac{\partial P_{L}}{\partial \omega}
$$

Alternately, subtracting Equation 14 from Equation 15 results in Equation 17, the final efficiency island condition on the power loss equation.

$$
0=Q \frac{\partial P_{L}}{\partial Q}-\omega \frac{\partial P_{L}}{\partial \omega}
$$

\section{A.A. Efficiency Islands for Positive Polynomial Loss Model}

Equations 14 through 17 constitute a set of conditions for the existence of efficiency islands given a power loss function of the form $P_{L}=f(\omega, Q)$. In Section III, Equation 8 was presented as a generalized power loss model for electric motors. Applying the conditions of Equations 14 through 17 to Equation 8 requires taking the partial derivatives of Equation 8 with respect to torque and motor speed. The partial derivative of Equation 8 with respect to torque is Equation 18.

$$
\frac{\partial P_{L}}{\partial Q}=\sum_{i=0}^{n} \sum_{j=0}^{m} C_{i, j} i Q^{i-1} \omega^{j} ; \quad \text { Where } C_{i, j} \geq 0
$$

Multiplying Equation 18 by torque elevates the exponent on torque from $i-1$ back to $i$ as shown in Equation 19.

$$
\begin{gathered}
Q \frac{\partial P_{L}}{\partial Q}=\sum_{i=0}^{n} \sum_{j=0}^{m} C_{i, j} i Q^{i} \omega^{j} ; \quad \text { Where } C_{i, j} \geq 0 \\
8 \text { of } 9
\end{gathered}
$$


Subtracting Equation 19 from Equation 8 yields Equation 20, which, when set equal to zero is the first efficiency island condition for a positive polynomial power loss equation.

$$
P_{L}-Q \frac{\partial P_{L}}{\partial Q}=\sum_{i=0}^{n} \sum_{j=0}^{m} C_{i, j}(1-i) Q^{i} \omega^{j} ; \quad \text { Where } C_{i, j} \geq 0
$$

These steps are repeated to arrive at Equation 21, which is the second efficiency island condition for a positive polynomial power loss equation.

$$
P_{L}-\omega \frac{\partial P_{L}}{\partial \omega}=\sum_{i=0}^{n} \sum_{j=0}^{m} C_{i, j}(1-j) Q^{i} \omega^{j} ; \quad \text { Where } C_{i, j} \geq 0
$$

Equations 20 and 21 must both equal zero for an efficiency island to be possible. The trivial solution where all coefficients $C_{i, j}=0$ corresponds to no power losses and is not a useful solution; some coefficient(s) must therefore be non-zero. Given that there are some positive terms in the sum, for the sum to equal zero, there must also be some negative terms. Because the coefficients are restricted to be non-negative, and we are only interested in positive values of torque and motor speed, the only way to arrive at a negative term in Equation 20 is for there to be some term with non-zero $C_{i, j}$ where $(1-i)<0$; this condition requires that $n \geq 2$. Applying the same procedure to Equation 21 requires that $m \geq 2$.

In other words, given a positive polynomial power loss model, in order for an efficiency island to exist, there must be terms of at least second order in torque and motor speed. We immediately can see that the standard equivalent circuit model discussed in Section II.B with $n=2$ and $m=1$ is not capable of representing an efficiency island.

Equations 20 and 21 are summed to result in Equation 22, the third efficiency island condition.

$$
2 P_{L}-Q \frac{\partial P_{L}}{\partial Q}-\omega \frac{\partial P_{L}}{\partial \omega}=\sum_{i=0}^{n} \sum_{j=0}^{m} C_{i, j}(2-i-j) Q^{i} \omega^{j} ; \quad \text { Where } C_{i, j} \geq 0
$$

Continuing the logic applied to the first two conditions reveals that in order for Equation 22 to equal zero, there must be some term with non-zero coefficient with $(2-i-j)<0$; this condition requires that $n+m \geq 3$.

In other words, given a positive polynomial power loss model, in order for an efficiency island to exist, there must be at least one term where the combined order of torque and speed is cubic.

Equation 20 is subtracted from Equation 21 to result in Equation 23, the fourth efficiency island condition. No useful conditions can be derived from Equation 23.

$$
Q \frac{\partial P_{L}}{\partial Q}-\omega \frac{\partial P_{L}}{\partial \omega}=\sum_{i=0}^{n} \sum_{j=0}^{m} C_{i, j}(i-j) Q^{i} \omega^{j} ; \quad \text { Where } C_{i, j} \geq 0
$$

Consideration of the loss buildup model presented in Section II.A with $n=2$ and $m=3$ in light of the conditions derived from Equations 20 through 22 suggest that the loss buildup model is capable of supporting an efficiency island.

\section{References}

1 "UQM PowerPhase 125," Specification Sheet, 2010.

${ }^{2}$ Larminie, J. and Lowry, J., Electric Vehicle Technology Explained, John Wiley \& Sons Ltd., 2nd ed., 2012.

${ }^{3}$ Gur, O. and Rosen, A., "Optimizing Electric Propulsion Systems for Unmanned Aerial Vehicles," Journal of Aircraft, Vol. 46, No. 4, July-August 2009, pp. 1340-1353.

${ }^{4}$ Stepaniak, M. J., van Graas, F., and de Haag, M. U., "Design of an Electric Propulsion System for a Quadrotor Unmanned Aerial Vehicle," Journal of Aircraft, Vol. 46, No. 3, May-June 2009, pp. 1050-1058.

${ }^{5}$ Drela, M., "Second-Order DC Electric Motor Model," March, 2006.

${ }^{6}$ Carri, J., "A four-constant model for electric motors," April 23, 2007.

${ }^{7}$ Lawson, C. L. and Hanson, R. J., Solving Least Square Problems, Prentice Hall, Englewood Cliffs, NJ, 1974, Chapter 23. 